PROCEEDINGS OF THE

AMERICAN MATHEMATICAL SOCIETY

Volume 131, Number 10, Pages 3163-3175

S 0002-9939(03)07107-7

Article electronically published on May 9, 2003

\title{
LINEAR CONTINUOUS DIVISION FOR EXTERIOR AND INTERIOR PRODUCTS
}

\author{
P. DOMAŃSKI AND B. JAKUBCZYK
}

(Communicated by Jozef Dodziuk)

Abstract. We consider the complex

$0 \longrightarrow \Lambda_{0}(M ; E) \stackrel{\partial_{\omega}}{\longrightarrow} \Lambda_{1}(M ; E) \stackrel{\partial_{\omega}}{\longrightarrow} \ldots \stackrel{\partial_{\omega}}{\longrightarrow} \Lambda_{m}(M ; E)$,

where $E$ is a finite-dimensional vector bundle over a suitable differential manifold $M, \Lambda_{q}(M ; E)$ denotes the space of all smooth or real analytic or holomorphic sections of the q-exterior product of $E$ and $\partial_{\omega}(\eta):=\omega \wedge \eta$ for $\omega \in \Lambda_{1}(M ; E)$. We give sufficient and necessary conditions for the above complex to be exact and, in smooth and holomorphic cases, we give sufficient conditions for its splitting, i.e., for existence of linear continuous right inverse operators for $\partial_{\omega}: \Lambda_{q}(M ; E) \rightarrow \operatorname{Im} \partial_{\omega} \subseteq \Lambda_{q+1}(M ; E)$.

Analogous results are obtained whenever $M$ is replaced by a suitable closed subset $X$ or $\partial_{\omega}$ are replaced by the interior product operators $\left.\partial_{Z}, \partial_{Z}(\eta):=Z\right\rfloor \eta$ for a given section $Z$ of the dual bundle $E^{*}$.

\section{INTRODUCTION}

This note is motivated by the following problem. Let $\omega$ and $\eta$ be, respectively, a differential 1 -form and a differential $k$-form on a smooth, paracompact manifold $M$ of dimension $n$. We ask two questions: (1) under what conditions does $\omega$ divide $\eta$, i.e. there exists a $(k-1)$-form $\gamma$ such that $\eta=\omega \wedge \gamma ;(2)$ can this division be done via a linear continuous (in the $C^{\infty}$ topology) operator, i.e., if there is such an operator which maps $\eta$ into $\gamma$ ?

These problems originate from the theory of differential forms and differential equations. One such question appears, for instance, when the Moser homotopy method is used in problems of equivalence of contact or symplectic structures with singularities (see [12, [13]). The crucial problem when applying this method is to solve the so-called homological equation. Our results give a tool for solving this equation and were already applied in [13.

To examine the problem (1) note that the obvious necessary condition on $\eta$ is $\omega \wedge \eta=0$. It is also sufficient, locally around regular points of $\omega$, and the difficulty of the first problem is reduced to points where $\omega$ vanishes. We use the notion of

Received by the editors May 7, 2002.

2000 Mathematics Subject Classification. Primary 46E10, 58A10.

Key words and phrases. Exact complexes, splitting, exterior and interior multiplication, division properties, spaces of smooth functions, spaces of holomorphic functions, spaces of real analytic functions.

The research of the second named author was partially supported by the Committee for Scientific Research, Poland, grant KBN 2P03A 03516. 
depth from commutative algebra (see e.g. [8]) which provides an adequate tool for stating restrictions on possible singularities of $\omega$ so that the necessary division condition also becomes sufficient for local and global divisibility (Theorem 2.1).

A more general version of the second problem, for trivial bundles, appears if we multiply sections of a bundle by a matrix with smooth coefficients. In this setting Bierstone and Schwartz [3, Th. 0.1.3] solved the second problem even on a large class of subanalytic closed subsets $X$ of $M$ instead of $M$ itself, assuming that the matrix has real analytic coefficients. A subproblem which appeared there was to extend Malgrange's Theorem on closedness of ideals generated by analytic functions. For a more general setting see [1].

We consider the problem for arbitrary smooth sections $\omega$ of a vector bundle and arbitrary closed sets. We give a condition on $\omega$ which implies that the necessary division condition $\omega \wedge \eta=0$ is also sufficient (Theorem 2.1) and, moreover, we show that the same condition is sufficient for the existence of "division operators" (Theorem 2.2). Then we provide some solutions to analogous problems in the real analytic category and in the holomorphic category (Theorem 2.1 and Theorem 2.3). Probably the main novelty and the core of the paper is to apply abstract functional analytic methods developed by Vogt and his collaborators (splitting theorems, compare Lemma 3.6) in Theorems 2.2 and 2.3. Some versions of Theorem 2.1 might have been known to specialists. (We have not found a suitable reference and, therefore, we provide a complete proof.)

A similar problem as above appears if we replace the 1 -form $\omega$ by a vector field $Z$ on $M$ and the operator of exterior multiplication by the operator of interior product $\eta \rightarrow Z\rfloor \eta$. We provide analogous solutions in this case.

In this paper we explain the related notions and results in detail in order to make the paper accessible for a wider audience.

\section{Statement of Results}

Let us formulate the problem in the general setting. We consider a vector bundle $E$ over $M$, of rank $m$, and denote by $E^{*}$ its dual. All objects, as well as morphisms, functions, sections, etc., will be considered in the category $C^{s}$, which is any of the three categories: $C^{\infty}$ (smooth), $C^{\omega}$ (real analytic), $C^{\text {Stein }}$ (holomorphic). In the last case we assume that $M$ is a complex Stein manifold. We denote by $\Lambda_{q}^{s}(M ; E)$ the linear space of $C^{s}$ sections of the $q$-th skew-symmetric power of $E$. In particular, $\Lambda_{0}^{s}(M)=\Lambda_{0}^{s}(M ; E)$ is the space of $C^{s}$ functions on $M$, also denoted by $C^{s}(M)$.

Any $C^{s}$ section $\omega$ of $E$ defines the linear operator of exterior multiplication by $\omega$, which gives the complex

$$
0 \longrightarrow \Lambda_{0}^{s}(M) \stackrel{\partial_{\omega}}{\longrightarrow} \Lambda_{1}^{s}(M ; E) \stackrel{\partial_{\omega}}{\longrightarrow} \cdots \stackrel{\partial_{\omega}}{\longrightarrow} \Lambda_{m}^{s}(M ; E)
$$

with the operator $\partial_{\omega}=\partial_{\omega}^{p}: \Lambda_{p}^{s}(M ; E) \rightarrow \Lambda_{p+1}^{s}(M ; E)$ defined by

$$
\partial_{\omega}^{p}(\gamma):=\omega \wedge \gamma
$$

Similarly, any $C^{s}$ section $Z$ of the dual bundle $E^{*}$ defines the operator of interior product $\left.\partial_{Z}=\partial_{Z}^{p}=Z\right\rfloor$ given on exterior products of sections $\omega_{1}, \ldots, \omega_{p}$ of $E$ by

$$
Z\rfloor\left(\omega_{1} \wedge \cdots \wedge \omega_{p}\right)=\sum_{i=1}^{p}(-1)^{i+1}\left\langle Z, \omega_{i}\right\rangle \omega_{1} \wedge \cdots \wedge \breve{\omega}_{i} \wedge \cdots \wedge \omega_{p}
$$


where $\langle\cdot, \cdot\rangle$ denotes the duality product between $E^{*}$ and $E$, and $\breve{\omega}_{i}$ means absence of $\omega_{i}$. This operator defines the complex

$$
0 \longrightarrow \Lambda_{m}^{s}(M ; E) \stackrel{\partial_{Z}}{\longrightarrow} \Lambda_{m-1}^{s}(M ; E) \stackrel{\partial_{Z}}{\longrightarrow} \cdots \stackrel{\partial_{Z}}{\longrightarrow} \Lambda_{0}^{s}(M) .
$$

More generally, we can define the above complexes on subsets of $M$. Consider a closed subset $X \subset M$ of category $C^{s}$, i.e. a subset which, locally, is a set of zeros of a family of functions of category $C^{s}$. We denote by $\Lambda_{q}^{s}(M, X ; E)$ the space of $C^{s}$ sections of the $q$-th skew-symmetric power of $E$ which vanish on $X$ (in particular, $\Lambda_{0}^{s}(M, X)=\Lambda_{0}^{s}(M, X ; E)$ is the space of $C^{s}$ functions on $M$, vanishing on $\left.X\right)$. We define the quotient spaces

$$
\Lambda_{0}^{s}(X)=\Lambda_{0}^{s}(M) / \Lambda_{0}^{s}(M, X), \quad \Lambda_{q}^{s}(X ; E)=\Lambda_{q}^{s}(M ; E) / \Lambda_{q}^{s}(M, X ; E), \quad q \geq 1 .
$$

Any element $\omega$ of $\Lambda_{1}^{s}(X ; E)$ defines the unique operator

$$
\partial_{\omega}^{p}: \Lambda_{p}^{s}(X ; E) \rightarrow \Lambda_{p+1}^{s}(X ; E)
$$

(the quotient of the operator of exterior multiplication) which gives the complex

$$
0 \longrightarrow \Lambda_{0}^{s}(X) \stackrel{\partial_{\omega}}{\longrightarrow} \Lambda_{1}^{s}(X ; E) \stackrel{\partial_{\omega}}{\longrightarrow} \cdots \stackrel{\partial_{\omega}}{\longrightarrow} \Lambda_{m}^{s}(X ; E) .
$$

Similarly, any element $Z$ of $\Lambda_{1}^{s}\left(X ; E^{*}\right)$ defines the corresponding operator

$$
\partial_{Z}^{p}: \Lambda_{p}^{s}(X ; E) \rightarrow \Lambda_{p-1}^{s}(X ; E)
$$

the quotient of the operator of interior product. These operators define the complex

$$
0 \longrightarrow \Lambda_{m}(X ; E) \stackrel{\partial_{Z}}{\longrightarrow} \Lambda_{m-1}(X ; E) \stackrel{\partial_{Z}}{\longrightarrow} \cdots \stackrel{\partial_{Z}}{\longrightarrow} \Lambda_{0}(X) .
$$

For more information on complexes like those above, called Koszul complexes, we refer the reader to 10 , Ch. 5.

Let $R_{x}(X)$ denote the ring of function germs of class $C^{s}$ on $X$, at the point $x \in X$. This means that $R_{x}(X)=C_{x}^{s}(M) / C_{x}^{s}(M, X)$, where $C_{x}^{s}(M)$ is the ring of function germs (at $x)$ on $M$, of class $C^{s}$, and $C_{x}^{s}(M, X)$ is its ideal of function germs which vanish on $X$. For elements $\omega \in \Lambda_{1}^{s}(X ; E)$ and $Z \in \Lambda_{1}^{s}\left(X ; E^{*}\right)$ we introduce the ideals

$$
I_{x}(\omega) \subset R_{x}(X) \quad \text { and } \quad I_{x}(Z) \subset R_{x}(X)
$$

of $R_{x}(X)$ generated by the coefficients of representatives $\tilde{\omega} \in \Lambda_{1}^{s}(M ; E)$ of $\omega$ and $\tilde{Z} \in \Lambda_{1}^{s}\left(M ; E^{*}\right)$ of $Z$, respectively, in some coordinate system on $M$ at $x$.

Let us recall the notion of the depth of an ideal. Let $R$ be a commutative ring with a unit. Given a proper ideal $I$ of $R$, we say that a sequence of elements $a_{1}, \ldots, a_{q}$ of $I$ is a regular sequence if $a_{i}$ is not a zerodivisor in $R /\left(a_{1}, \ldots, a_{i-1}\right)$ for $i=1, \ldots, q$ (in particular, $a_{1}$ is not a zerodivisor in $R$ ). Here we denote by $\left(a_{1}, \ldots, a_{i}\right)$ the ideal in $R$ generated by the elements $a_{1}, \ldots, a_{i}$. The depth of a proper ideal $I \subset R$, denoted depth $I$, is defined as the supremum of lengths of regular sequences in $I$. Additionally, one defines depth $R=\infty$. For additional information concerning the depth we refer the reader to [8] or [12], Appendix 1.

Remark. In the holomorphic category the depth of an ideal of holomorphic function germs coincides with the codimension of the analytic set germ of zeros of this ideal; it is therefore a computable invariant (see [10], Chapter 5.3). In the real analytic category the depth of an ideal coincides with the codimension of the set of its complex zeros since complexification does not change the depth; see [20]. 
We denote $\operatorname{Sing}(\omega)=\{x \in X: \omega(x)=0\}$ and $\operatorname{Sing}(Z)=\{x \in X: Z(x)=0\}$. The following theorem holds in the categories $C^{\infty}, C^{\omega}$, and $C^{\text {Stein }}$.

Theorem 2.1. Consider a closed subset $X \subset M$ which is additionally assumed to be real analytic and coherent, in the case of category $C^{\omega}$, and complex analytic, in the case of category $C^{\text {Stein }}$.

(a) If $\operatorname{depth} I_{x}(\omega) \geq k+1$ for all $x \in \operatorname{Sing}(\omega)$, then the complex (3) is exact up to $\Lambda_{k}^{s}(X ; E)$. Similarly, if $\operatorname{depth} I_{x}(Z) \geq k+1$ for all $x \in \operatorname{Sing}(Z)$, then the complex (4) is exact up to $\Lambda_{m-k}^{s}(X ; E)$.

(b) The converse statements hold in the categories $C^{\omega}$ and $C^{\text {Stein }}$.

We recall that a sheaf $\mathcal{F}$ of modules over $X$ (over a sheaf of rings $\mathcal{R}$ ) is called coherent if it satisfies two conditions: (a) it is of finite type (i.e., locally, it is generated by a finite number of sections); (b) given any sections $s_{1}, \ldots, s_{k}$ of $\mathcal{F}$ over an open $U \subset X$, the sheaf of relations, i.e. of the elements $\left(f_{1}, \ldots, f_{k}\right) \in(\mathcal{R}(U))^{k}$ such that $f_{1} s_{1}+\cdots+f_{k} s_{k}=0$, is of finite type.

An analytic subset $X \subset M$ of a real analytic (respectively, holomorphic, Stein) manifold $M$ is called coherent if the sheaf $\mathcal{O}(M, X)$ of real analytic (respectively, holomorphic) function germs vanishing on $X$ is coherent (over the sheaf $\mathcal{O}$ ). This is equivalent to saying that the sheaf of function germs vanishing on $X$ is of finite type (the sheaf of relations is always of finite type). All analytic subsets of Stein manifolds as well as every real analytic manifold are coherent by a theorem of Oka (see 4, Prop. 4]; for more information on coherence and real analytic coherent sets see [17]).

Remarks. (a) Statement (a) in Theorem 2.1 holds in the $C^{\infty}$ category if we assume that $M=X, \omega$ and $Z$ are real analytic and the depth used in the assumptions is computed in the ring of real analytic function germs $C_{x}^{\omega}(M)$ (equivalently, in the ring of formal power series $\mathcal{F}$ ). This follows from our proof where the depth assumption is used in Lemma 3.1 only, in order to show that the complexes (3) and (4) are locally exact. Namely, the depth assumption in the ring $C_{x}^{\omega}(M)$ guarantees local exactness of the complexes (3) and (4) in the real analytic category and the fact that the ring $C_{x}^{\infty}(M)$ is flat over $C_{x}^{\omega}(M)$ (cf. [14]) implies that the same complex is locally exact in the smooth category. Similarly, the depth assumption in the ring of formal power series $\mathcal{F}$ and faithful flatness of this ring over $C_{x}^{\omega}(M)$ (see 14]) guarantee local exactness of (3) and (4) in the real analytic category and so in the smooth category. Computing the depth of an ideal in the ring $\mathcal{F}$ can be much simpler than in the ring of smooth function germs.

(b) The maximal length of a regular sequence in an ideal $I$ cannot exceed the minimal number of its generators. Therefore, if $X=M^{d}$ and $\omega(0)=0, Z(0)=0$, then the ideals $I_{x}(\omega)$ and $I_{x}(Z)$ are contained in the maximal ideal of function germs vanishing at $x$, which has $d$ generators. It follows that the depth of these ideals does not exceed $\min \{d, m\}$. Therefore, if $d=m$, then the sequence (1) can be exact up to $\Lambda_{m-1}^{s}(M ; E)$. It cannot be exact at $\Lambda_{m}^{s}(M ; E)$, when prolonged by $\Lambda_{m}^{s}(X ; E) \rightarrow 0$, if $\omega(x)=0$ at some $x$ (easy to see).

(c) Local results similar to those stated in Theorem [2.1 were already used in solving linear singular equations concerning differential forms; cf. e.g. [16] and [12]. A global topological version stated in Theorem 2.2 below was applied in [13] for solving the so-called homological equation. 
In order to state our continuity result on division via operators we endow the spaces $\Lambda_{r}^{\infty}(M ; E)$ with the natural $C^{\infty}$ topology so that they become Fréchet spaces; $\Lambda_{r}^{\infty}(M, X ; E)$ denotes their closed subspaces and

$$
\Lambda_{r}^{\infty}(X ; E)=\Lambda_{r}^{\infty}(M ; E) / \Lambda_{r}^{\infty}(M, X ; E)
$$

are quotient Fréchet spaces.

Below we say that a complex of linear topological spaces and continuous linear operators

$$
0 \longrightarrow L_{0} \stackrel{T_{0}}{\longrightarrow} L_{1} \stackrel{T_{1}}{\longrightarrow} \cdots \longrightarrow L_{k} \stackrel{T_{k}}{\longrightarrow} L_{k+1}
$$

splits up to $L_{k}$ if each operator $T_{i-1}: L_{i-1} \rightarrow \operatorname{Im} T_{i-1} \subseteq L_{i}, i=1, \ldots, k$, has a continuous linear right inverse operator defined on the image (here $\operatorname{Im} T_{i}$ is equipped with the subspace topology induced from $L_{i+1}$ ). For other functional analytic notions and results we refer the reader to [15].

We say that a subset $X \subset M$ has the extension property (in the category $C^{\infty}$ ) if there exists a linear continuous operator $\lambda: C^{\infty}(X) \rightarrow C^{\infty}(M)$ such that $\lambda(f) \mid X=$ $f$ for all $f \in C^{\infty}(X)$, or more precisely, the natural quotient map $C^{\infty}(M) \rightarrow$ $C^{\infty}(X)$ has a linear continuous right inverse.

We have the following result.

Theorem 2.2. Let $M$ be $C^{\infty}$ manifold and let $X$ be a closed subset of $M$ which has the extension property. If depth $I_{x}(\omega) \geq k+1$ for all $x \in \operatorname{Sing}(\omega)$, then the complex (3) in the $C^{\infty}$ category splits up to $\Lambda_{k}^{\infty}(X ; E)$. Similarly, If $\operatorname{depth} I_{x}(Z) \geq k+1$ for all $x \in \operatorname{Sing}(Z)$, then (4) splits up to $\Lambda_{m-k}^{\infty}(X ; E)$.

Remarks. (a) It is known that for $M$ real analytic any closed semianalytic subset $X$ as well as any coherent closed subanalytic subset has the extension property. More extension results for subanalytic sets are contained in [2, Th. 1.23], [3, 0.2.1]. For general sets see [22], [9].

(b) If $E$ is a trivial bundle, $M, \omega, Z$ are real analytic and $X \subseteq M$ is a closed semicoherent subanalytic (in particular, semianalytic or subanalytic and coherent) subset, then the operators

$$
\partial_{\omega}: \Lambda_{p}^{\infty}(X ; E) \rightarrow \operatorname{Im} \partial_{\omega} \subseteq \Lambda_{p+1}^{\infty}(X ; E)
$$

and

$$
\partial_{Z}: \Lambda_{p+1}^{\infty}(X ; E) \rightarrow \operatorname{Im} \partial_{Z} \subseteq \Lambda_{p}^{\infty}(X ; E)
$$

have continuous linear right inverses even if the complexes are not exact! See 3 . Cor. 8.4], [2, Th. 1.13, Th. 1.23].

In the complex case we have to restrict the class of manifolds. We call a Stein manifold $M$ strongly pseudoconvex if there exists a strictly negative, vanishing at infinity plurisubharmonic function $u$ on $M$. We say that a Stein manifold $M$ satisfies the strong Liouville property if and only if every bounded above plurisubharmonic function on $M$ is constant on every connected component.

We denote by $H(M)$ the space of holomorphic functions on $M$ with the compactopen topology. Let us recall that, by [25, Th. 0.3], for a Stein manifold $M$ with finitely many connected components we have $H(M) \simeq H\left(\mathbb{C}^{d}\right)$ if and only if $\operatorname{dim} M=d$ and $M$ satisfies the strong Liouville property. Analogously, we have $H(M) \simeq H\left(\mathbb{D}^{d}\right)$ if and only if $\operatorname{dim} M=d$ and $M$ is strongly pseudoconvex (here $\mathbb{D}$ denotes the unit disc in $\mathbb{C}$ ). We equip $\Lambda_{k}^{\text {Stein }}(M ; E)$ with the compact-open topology so that they become Fréchet spaces. 
Theorem 2.3. Let $X=M$ be a Stein manifold with a finite number of connected components which is either strongly pseudoconvex or satisfies the strong Liouville property, and let $E$ be a trivial vector bundle over $M$ of rank $m$. Then the sequence (1) (resp. (2)) in the Stein category splits up to $\Lambda_{k}^{\text {Stein }}(M ; E)$ (up to $\Lambda_{n-k}^{\text {Stein }}(M ; E)$, resp. $)$, provided that $\operatorname{depth} I_{x}(\omega) \geq k+1$ for all $x \in \operatorname{Sing}(\omega)$ (respectively, $\operatorname{depth} I_{x}(Z) \geq k+1$ for all $x \in \operatorname{Sing}(Z)$ ).

Let us remark that $\mathbb{D} \times \mathbb{C}$ is a simplest example of a Stein manifold not covered by the result above.

Problem 2.4. Does Theorem 2.3 hold for arbitrary Stein manifolds $M$ and arbitrary analytic subsets $X$ in $M$ ?

\section{Proofs}

First, we need a result from commutative algebra. Let $R$ be a commutative ring with unity and let $Z=\left(a_{1}, \ldots, a_{m}\right)$ be an element of the free module $R^{m}$. We denote by $\Lambda_{k}\left(R^{m}\right)$ the $k$-th exterior power of $R^{m}$. Let $I$ be the ideal in $R$ generated by the coefficients of $Z$. Consider the complexes:

$$
0 \rightarrow R \rightarrow R^{m}=\Lambda_{1}\left(R^{m}\right) \rightarrow \cdots \rightarrow \Lambda_{m}\left(R^{m}\right),
$$

defined by the operators $Z \wedge$ of exterior multiplication by $Z$, and

$$
0 \rightarrow \Lambda_{m}\left(R^{m}\right) \rightarrow \Lambda_{m-1}\left(R^{m}\right) \rightarrow \cdots \rightarrow \Lambda_{1}\left(R^{m}\right) \rightarrow R,
$$

defined by the operators $Z\rfloor$ of interior product with $Z$ given as

$$
Z\rfloor\left(\omega_{1} \wedge \cdots \wedge \omega_{p}\right)=\sum_{i=1}^{p}(-1)^{i+1}\left\langle Z, \omega_{i}\right\rangle \omega_{1} \wedge \cdots \wedge \breve{\omega}_{i} \wedge \cdots \wedge \omega_{p}
$$

$\left(\breve{\omega}_{i}\right.$ means omission of $\omega_{i}$ and $\langle Z, \omega\rangle=\sum_{i} a_{i} b_{i}$, for $\left.\omega=\left(b_{1}, \ldots, b_{m}\right)\right)$. Denote by $I=I(Z)$ the ideal generated by $a_{1}, \ldots, a_{m}$. The following result is classic (see e.g. [8]).

Lemma 3.1. If depth $I \geq k+1$, then the first complex is exact up to $\Lambda_{k}\left(R^{m}\right)$ and the second is exact up to $\Lambda_{m-k}\left(R^{n}\right)$. If $R$ is noetherian, then the converse implications hold, too.

Lemma 3.1 and the following lemma which holds in the categories $C^{\infty}, C^{\omega}$, and $C^{\text {Stein }}$ implies Theorem 2.1 since the rings of germs of real or complex analytic functions are noetherian.

Lemma 3.2. Let $X \subset M$ be a closed subset which is additionally real analytic and coherent (in the $C^{\omega}$-case) or complex analytic (in the $C^{\text {Stein }}$-case). Then the complex (3) (respectively, (4)) is exact at $\Lambda_{r}^{s}(X ; E)$ if and only if it is exact locally at $\Lambda_{r}^{s}(X ; E)$, i.e. for germs at any $p \in X$.

Proof. We shall prove the lemma in the case of complex (4) (the proof in the case of complex (3) is analogous).

Assume that (4) is locally exact at $\Lambda_{r}$ at any point $p \in X$, and also at any $p \in M \backslash X$ (where this property is trivial). For the sake of brevity we denote $\Lambda_{r}(X)=\Lambda_{r}^{s}(X ; E), \Lambda_{r}(M)=\Lambda_{r}^{s}(M ; E)$, and $\Delta_{r}(M)=\Lambda_{r}^{s}(M, X ; E)$, adding the subscript $X_{p}$ or $M_{p}$ if we mean germs at $p$. Then $\Lambda_{r}(X)=\Lambda_{r}(M) / \Delta_{r}(M)$.

In order to show that (4) is globally exact at $\Lambda_{r}$ we assume that $\nu \in \Lambda_{r}(M)$ and $Z\rfloor \nu=0$ in $\Lambda_{r-1}(X)$, i.e., $\left.Z\right\rfloor \nu=0$ modulo $\Delta_{r-1}(M)$. Here and below we shall 
denote by the same letter $Z$ the element of $\Lambda\left(X ; E^{*}\right)=\Lambda\left(M ; E^{*}\right) / \Lambda\left(M, X ; E^{*}\right)$ (the equivalence class) defining the complex (4) or a (fixed during the proof) representative in $\Lambda\left(M ; E^{*}\right)$ of this equivalence class. We should show that there are $\mu \in \Lambda_{r+1}(M)$ and $\eta \in \Delta_{r+1}(M)$ such that $\left.\nu=Z\right\rfloor \mu+\eta$. At any point $p \in M$ we have the germs equality: $\left.Z_{p}\right\rfloor \nu_{p}=0$ modulo $\Delta_{r-1}\left(M_{p}\right)$. The local exactness of the complex at $\Lambda_{r}$ implies that there exists a germ $\mu_{p} \in \Lambda_{r+1}\left(M_{p}\right)$ such that $\left.Z_{p}\right\rfloor \mu_{p}=\nu_{p}$ modulo $\Delta_{r}\left(M_{p}\right)$. Replacing the germs by objects defined on open sets $U_{i}$, with $\bigcup_{i} U_{i}=M$, we have

$$
Z\rfloor \mu_{i}=\nu+\eta_{i} \quad \text { on } U_{i},
$$

where $\eta_{i}$ is a $C^{s}$ section of $\Lambda_{r}$ defined on $U_{i}$ and $\left.\eta\right|_{X \cap U_{i}}=0$. In the $C^{\infty}$ category we use a smooth partition of unity $\left\{\psi_{i}\right\}$ corresponding to the covering $\left\{U_{i}\right\}$ of $M$. We put $\mu=\sum_{i} \psi_{i} \mu_{i}$ and $\eta=\sum_{i} \psi_{i} \eta_{i}$; then $\left.\eta\right|_{X}=0$ and we get $\left.Z\right\rfloor \mu=$ $\sum_{i} \psi_{i} \nu+\sum_{i} \psi_{i} \eta_{i}=\nu+\eta$. This means that the global complex (4) is exact at $\Lambda_{r}$.

In the $C^{\omega}$ and $C^{\text {Stein }}$ categories we shall use the real-analytic and holomorphic versions of Cartan's Theorem B, instead of partition of unity.

Let us fix the category $C^{\omega}$. Denote by $\mathcal{O}(M)$ and $\mathcal{O}(X)$ the sheaves of germs of real analytic functions on $M$ and $X$, respectively. Then $\mathcal{O}(X)$ is the quotient sheaf $\mathcal{O}(M) / \mathcal{O}(M, X)$ (with $\mathcal{O}(M, X)$ the subsheaf of real analytic functions vanishing on $X)$ and so it is coherent as the quotient of two coherent sheaves. Let $\mathcal{O}\left(X, \Lambda_{r}\right)$ denote the sheaf of germs of real analytic sections over $X$ of the $r$-th skew-symmetric product of $E$ (any such germ at $p \in X$ is, by definition, a restriction to $X$ of a germ of an analytic section on $M)$. Then, locally, $\mathcal{O}\left(X, \Lambda_{r}\right)$ is isomorphic to the sheaf $(\mathcal{O}(U))^{k}$, where $U \subset X$ is open and $k$ is a positive integer, and so it is coherent. The operator $Z\rfloor$ defines a morphism of coherent sheaves $\mathcal{O}\left(X, \Lambda_{r+1}\right) \rightarrow \mathcal{O}\left(X, \Lambda_{r}\right)$. The kernel subsheaf $\operatorname{ker}(Z\rfloor)$ of $\mathcal{O}\left(M, \Lambda_{r+1}\right)$ is coherent (Theorem 2, Section 13 in 21]).

Having the earlier formula $Z\rfloor \mu_{i}=\nu+\eta_{i}$ on $U_{i}$, with $\left.\eta_{i}\right|_{X \cap U_{i}}=0$, we can write $Z\rfloor \mu_{i}=\nu$ on $X \cap U_{i}$. We define the collection of sections of $\mathcal{O}\left(X, \Lambda_{r+1}\right)$ on $U_{i} \cap U_{j} \cap X$ as

$$
\gamma_{i j}=\mu_{i}-\mu_{j},
$$

which form a 1-cocycle on $X$ with values in the sheaf $\mathcal{F}=\operatorname{ker}(Z\rfloor$ ) (since $Z\rfloor\left(\mu_{i}-\mu_{j}\right)=\nu-\nu=0$ on $\left.U_{i} \cap U_{j} \cap X\right)$. Cartan's Theorem B [11, III.3.7] (compare [4, Th. 3] and the Grauert embedding theorem for real analytic manifolds) implies that the cohomology groups $H^{q}(X, \mathcal{F})$ vanish for $q \geq 1$ and $\mathcal{F}$ a coherent sheaf of modules over the coherent sheaf of rings of real analytic functions on $X$ (which is a coherent analytic subset of $M$ ). Taking $\mathcal{F}=\operatorname{ker}(Z\rfloor)$ it follows, after a possible subdivision of the covering, that there exist elements $\gamma_{i}$ (local sections of $\mathcal{F}$ ) defined on $U_{i} \cap X$ such that $\left.Z_{i}\right\rfloor \gamma_{i}=0$ and

$$
\gamma_{i j}=\gamma_{i}-\gamma_{j}
$$

on $U_{i} \cap U_{j} \cap X$. We define

$$
\tilde{\mu}_{i}=\mu_{i}-\gamma_{i} .
$$

Then $\tilde{\mu}_{i}-\tilde{\mu}_{j}=\mu_{i}-\mu_{j}-\gamma_{i j}=0$ on $U_{i} \cap U_{j} \cap X$. It follows that $\tilde{\mu}_{i}=\tilde{\mu}_{j}$ on $U_{i} \cap U_{j} \cap X$ and the section $\mu_{X}$ given by

$$
\left.\mu_{X}\right|_{U_{i}}=\tilde{\mu}_{i}
$$

is correctly defined and of class $C^{\omega}$ on $X$. We have $\left.\left.(Z\rfloor \mu_{X}\right)\left.\right|_{U_{i} \cap X}=Z\right\rfloor \tilde{\mu}_{i}=$ $\left.Z\rfloor \mu_{i}-Z\right\rfloor \gamma_{i}=\left.\nu\right|_{U_{i} \cap X}$, and so $\left.Z\right\rfloor \mu_{X}=\nu$ on $X$. 
In order to complete the proof we should show that the section $\mu_{X}$ has an extension to $M$ having the required properties. Let us replace the local sections $\tilde{\mu}_{i}$ which were defined above on $X$ by some local sections on $M$, denoted again $\tilde{\mu}_{i}$, defined on open subsets $U_{i}$ of $M$. We add the additional open subset $U_{0}=M \backslash X$ so that we obtain the covering of $M$ by open sets $U_{i}$. We define $\mu_{0}=0$ and $\gamma_{0}=0$. Define the cocycle

$$
\beta_{i j}=\tilde{\mu}_{i}-\tilde{\mu}_{j}
$$

with values in the sheaf $\mathcal{G}=\mathcal{O}\left(M, X ; \Lambda_{r+1}\right)$ of sections of the $r+1$ skew-symmetric product of $E$, vanishing on $X$. The sheaf $\mathcal{G}$ is coherent, as a subsheaf of finite type of the coherent sheaf $\mathcal{O}\left(M, \Lambda_{r+1}\right)$, thus the first cohomology group with values in $\mathcal{G}$ vanishes ([11, III.3.7]). Thus there are sections $\beta_{i}$ of $\mathcal{G}$ defined on $U_{i}$ such that $\beta_{i j}=\beta_{i}-\beta_{j}$ on $U_{i} \cap U_{j}$. We define a global section $\mu$ of $\mathcal{O}\left(M, \Lambda_{r+1}\right)$ via

$$
\left.\mu\right|_{U_{i}}=\tilde{\mu}_{i}-\beta_{i} .
$$

By the definition of $\beta_{i}$ this section is well defined on $M$ and it equals $\mu_{X}$, when restricted to $X$. Thus $Z\rfloor \mu=\nu$ on $X$ and so $Z\rfloor \mu-\nu=0$ modulo $\mathcal{O}\left(M, X ; \Lambda_{r}\right)$. This completes the proof that local exactness implies global exactness in the real analytic category.

The same argument works in the category $C^{\text {Stein }}$, where we use the holomorphic version of Cartan's Theorem B (Théorème fondamental in [4]).

To show that global exactness implies local exactness (in the categories $C^{\omega}$ and $\left.C^{\text {Stein }}\right)$ we consider a germ $\nu_{p} \in \Lambda_{r}(X)$ such that $\left.Z_{p}\right\rfloor \nu_{p}=0$. Since the subsheaf $\mathcal{G}=\operatorname{ker}(Z\rfloor)$ of $\mathcal{O}\left(X, \Lambda_{r}\right)$ is coherent, it follows from [11, III.3.7] (compare Theorem A of Cartan [4] and the Grauert embedding theorem) that $\nu_{p}$ belongs to the module generated by global sections of $\mathcal{G}$. Thus $\nu_{p}=\sum \phi_{p}^{i} \nu_{p}^{i}$, with $\nu^{i}$-global sections of $\mathcal{G}$. By exactness of the global complex we have $\left.\nu^{i}=Z\right\rfloor \mu^{i}$ and so $\left.\nu_{p}=Z\right\rfloor\left(\sum \phi_{p}^{i} \mu_{p}^{i}\right)$, which proves exactness of the local complex. In the category $C^{\infty}$ the proof goes by replacing a representative $\nu$ of a germ $\nu_{p}$ satisfying $\left.Z_{p}\right\rfloor \nu_{p}=0$ by $\tilde{\nu}=\psi \nu$ and so getting a global section satisfying $Z\rfloor \tilde{\nu}=0$, where $\psi$ is a smooth "cut-off" function on $M(\psi \equiv 1$ in some neighbourhood of $p$ and vanishes outside of a larger neighbourhood). The proof is complete.

We shall prove Theorems 2.2 and 2.3 using the same method and reducing them to abstract splitting theorems in the categories of "graded" and "tame" Fréchet spaces, respectively.

By $\Lambda_{r}(\alpha)$ we denote finite type $(r=0)$ and infinite type $(r=\infty)$ power series spaces,

$$
\begin{aligned}
\Lambda_{0}(\alpha) & :=\left\{x=\left(x_{n}\right)_{n \in \mathbb{N}} \in \mathbb{C}^{\mathbb{N}}: \forall k\|x\|_{k}:=\sup _{n \in \mathbb{N}}\left|x_{n}\right| e^{\left(-\frac{1}{k}\right) \alpha_{n}}<\infty\right\}, \\
\Lambda_{\infty}(\alpha) & :=\left\{x=\left(x_{n}\right)_{n \in \mathbb{N}} \in \mathbb{C}^{\mathbb{N}}: \forall k\|x\|_{k}:=\sup _{n \in \mathbb{N}}\left|x_{n}\right| e^{k \alpha_{n}}<\infty\right\},
\end{aligned}
$$

where $\alpha=\left(\alpha_{n}\right)_{n \in \mathbb{N}}$ is an increasing sequence of positive numbers tending to infinity (see [15, §29]). The space $s$ of rapidly decreasing sequences is defined to be $\Lambda_{\infty}(\log n)$.

It is known that $H\left(\mathbb{C}^{d}\right) \simeq \Lambda_{\infty}\left(n^{1 / d}\right)$ and $H\left(\mathbb{D}^{d}\right) \simeq \Lambda_{0}\left(n^{1 / d}\right)$ (see [19, Cor. 8.3.2]).

Now, we introduce "tame" notions - all of them are described in [18] but for the sake of completeness we explain some of them here. Let us recall that an operator $T: E \rightarrow F$, where $\left(E,\left(\|\cdot\|_{n}\right)_{n \in \mathbb{N}}\right)$ and $\left(F,\left(\|\cdot\|_{n}\right)_{n \in \mathbb{N}}\right)$ are Fréchet spaces equipped 
with the fixed sequences of seminorms, is called tame if there is a $p \in \mathbb{N}$ and a sequence of positive constants $\left(c_{n}\right)_{n \in \mathbb{N}}$ such that

$$
\|T x\|_{n} \leq c_{n}\|x\|_{n+p} \quad \text { for every } x \in E, n \in \mathbb{N} .
$$

We define the "tame category" where objects are Fréchet spaces equipped with fixed families of seminorms defining their topologies, and morphisms are linear tame operators. The spaces $\left(E,\left(\|\cdot\|_{n}\right)_{n \in \mathbb{N}}\right),\left(F,\left(\|\cdot\|_{n}\right)_{n \in \mathbb{N}}\right)$ are tamely isomorphic whenever there is an isomorphism $T: E \rightarrow F$ such that both $T$ and $T^{-1}$ are tame. A sequence

$$
\left(E_{1},\left(\|\cdot\|_{n}\right)_{n \in \mathbb{N}}\right) \stackrel{T_{1}}{\longrightarrow}\left(E_{2},\left(\|\cdot\|_{n}\right)_{n \in \mathbb{N}}\right) \stackrel{T_{2}}{\longrightarrow}\left(E_{3},\left(\|\cdot\|_{n}\right)_{n \in \mathbb{N}}\right)
$$

is tamely exact at $E_{2}$ if $\operatorname{ker} T_{2}=\operatorname{Im} T_{1}, T_{1}, T_{2}$ are tame and there is a $p \in \mathbb{N}$ and a sequence of positive constants $\left(C_{n}\right)_{n \in \mathbb{N}}$ such that the image via $T_{1}$ of the unit ball of the $n$-th seminorm on $E_{1}$ contains the intersection of $\operatorname{Im} T_{1}$ and the ball of radius $C_{n}$ of the $(n+p)$-th seminorm on $E_{2}$. For the definitions of other "tame notions" see [18].

The recalled power series spaces equipped with the above given sequence of norms play an important role in the tame category. By [25, Th. 3.3.1 and the proof on p. 133], we have the following result:

Lemma 3.3. If $M, \operatorname{dim} M=d$, is a strongly pseudoconvex Stein manifold with finitely many connected components, then for some plurisubharmonic strictly negative vanishing at infinity function $\Phi$ on $M$ the space $\left(H(M),\left(\|\cdot\|_{n}\right)_{n \in \mathbb{N}}\right)$ is tamely isomorphic to $\Lambda_{0}\left(n^{1 / d}\right)$, where

$$
\|f\|_{n}:=\sup \left\{|f(z)|: \Phi(z)<-\frac{1}{n}\right\}
$$

and

$$
\Omega_{c}:=\{z \in M: \Phi(z)<c\}, \quad c<0,
$$

is a relatively compact exhaustion of $M$.

Clearly, the sets $\Omega_{c}$ are also Stein manifolds.

The "graded notions" are all explained in [5]. For the sake of completeness we explain some of them now. Every object of the "graded category" (called a graded Fréchet space) consists of a Fréchet space $X$ and increasing sequence $\left(\tau_{n}\right)_{n \in \mathbb{N}}$ of semimetrizable linear topologies on $X$ whose union is equal to the original topology of $X$. A linear continuous operator $T:\left(X,\left(\tau_{n}\right)_{n \in \mathbb{N}}\right) \rightarrow\left(Y,\left(\tau_{n}\right)_{n \in \mathbb{N}}\right)$ is a graded operator whenever for every $n$ there is $m$ such that $T:\left(X, \tau_{m}\right) \rightarrow\left(Y, \tau_{n}\right)$ is continuous. The operator $T$ is a graded isomorphism if it is a linear isomorphism and both $T$ and $T^{-1}$ are graded operators. A sequence of graded spaces and graded operators

$$
\left(E_{1},\left(\tau_{n}\right)_{n \in \mathbb{N}}\right) \stackrel{T_{1}}{\longrightarrow}\left(E_{2},\left(\tau_{n}\right)_{n \in \mathbb{N}}\right) \stackrel{T_{2}}{\longrightarrow}\left(E_{3},\left(\tau_{n}\right)_{n \in \mathbb{N}}\right)
$$

is graded exact at $E_{2}$ if $\operatorname{ker} T_{2}=\operatorname{Im} T_{1}$ and for every $n$ there is $m$ such that the $T_{1}$-image of every $\tau_{n}$-0-neighbourhood in $E_{1}$ contains an intersection of a $\tau_{m}-0$ neighbourhood in $E_{2}$ with $\operatorname{Im} T_{1}$.

The special role in the graded category is played by the graded Fréchet space $g-s^{\mathbb{N}}$. As a Fréchet space it is a countable product of the spaces of rapidly decreasing sequences $s$. Its grading is given by the sequence of topologies induced via projections onto finite subproducts. If $M$ is a smooth manifold, then we take a 
sequence $\left(\Omega_{n}\right)_{n \in \mathbb{N}}, \Omega_{0} \subset \subset \Omega_{1} \subset \subset \cdots \subset \subset M, \bigcup \Omega_{n}=M$. We equip $C^{\infty}(M)$ with a grading $\left(\tau_{n}\right)_{n \in \mathbb{N}}$, where $\tau_{n}$ is induced from the standard topology of $C^{\infty}\left(\Omega_{n}\right)$. The gradings obtained from different sequences $\left(\Omega_{n}\right)_{n \in \mathbb{N}}$ are all equivalent.

Lemma 3.4. Let $M$ be a non-compact $C^{\infty}{ }_{\text {-manifold; then }} C^{\infty}(M)$ is graded isomorphic to $g-s^{\mathbb{N}}$. If $X$ has the extension property, then $C^{\infty}(X)$ is graded isomorphic to a graded complemented subspace of $C^{\infty}(M)$ and, thus, graded isomorphic to a graded complemented subspace of $g-s^{\mathbb{N}}$. The same holds for $C^{\infty}(X ; E)$, where $E$ is a finite-dimensional vector bundle over $M$.

Proof. Graded isomorphism of $C^{\infty}(M)$ and $C^{\infty}(M ; E)$ with $g-s^{\mathbb{N}}$ is proved in [5. Th. 2.4].

Let $S: C^{\infty}(X) \rightarrow C^{\infty}(M)$ be an extension map. Let $\left(U_{i}\right)_{i \in I}$ be a locally finite open covering of $X$ and let $\left(V_{i}\right)_{i \in I}$ be a locally finite family of open sets in $M$ such that $U_{i} \subset \subset V_{i} \subset \subset M$. Let $\left(\varphi_{i}\right)_{i \in I}$ be a smooth partition of unity on $X$ inscribed in $\left(U_{i}\right)_{i \in I}$. Moreover, let $\left(\psi_{i}\right)_{i \in I}$ be a family of smooth functions, $0 \leq \psi_{i} \leq 1$, $\operatorname{supp} \psi_{i} \subseteq V_{i},\left.\psi_{i}\right|_{U_{i}} \equiv 1$. We define

$$
T: C^{\infty}(X) \rightarrow C^{\infty}(M), \quad T(f):=\sum_{i} \psi_{i} S\left(f \varphi_{i}\right) .
$$

It is easily seen that $T$ is an extension operator and that it is graded for the natural gradings in $C^{\infty}(X)$ and $C^{\infty}(M)$. Since the restriction map $R: C^{\infty}(M) \rightarrow C^{\infty}(X)$ is graded and the identity map id : $C^{\infty}(X) \rightarrow C^{\infty}(X)$ factorizes as id $=R \circ T$, then $C^{\infty}(X)$ is graded isomorphic to a graded complemented subspace of $C^{\infty}(M)$.

Analogous arguments work in the case $C^{\infty}(X ; E)$.

Now, we summarize some splitting results. We start with the following wellknown lemma.

Lemma 3.5. If E, $F$ are Fréchet spaces such that every short exact sequence of Fréchet spaces

$$
0 \longrightarrow E \longrightarrow X \longrightarrow F
$$

splits, then for every complemented subspace $E_{1} \subseteq E, F_{1} \subseteq F$ every short exact sequence of Fréchet spaces

$$
0 \longrightarrow E_{1} \stackrel{j_{1}}{\longrightarrow} G \stackrel{q_{1}}{\longrightarrow} F_{1} \longrightarrow 0
$$

also splits. Analogous results hold in the graded category and in the tame category.

Proof. We create a commutative diagram with exact rows, where $E=E_{1} \oplus E_{2}$ and $F=F_{1} \oplus F_{2}$ :

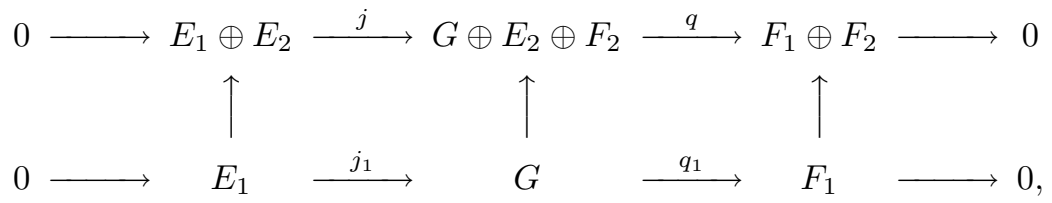

where we define $j\left(e_{1}, e_{2}\right):=\left(j_{1} e_{1}, e_{2}, 0\right), q\left(x, e_{2}, f_{2}\right):=\left(q_{1} x, f_{2}\right)$. Let $R$ be a right continuous linear inverse for $q$, and let $P: G \oplus E_{2} \oplus F_{2} \rightarrow G$ be the natural projection. Then $R_{1}: F_{1} \rightarrow G, R_{1}=\left.P \circ R\right|_{F_{1}}$, is a right continuous linear inverse of $q_{1}$.

Of course, the same method works in the graded and in the tame categories. 
The following lemma summarizes consequences of the three known splitting theorems $([15, \S 30],[5,18])$ and plays the crucial role in the proof of the continuous linear division results. An analogous theorem is also true when the $X_{i}$ are isomorphic to spaces of distributions (see [6], [7]).

Lemma 3.6. (a) Every exact complex

$$
0 \longrightarrow X_{0} \stackrel{T_{0}}{\longrightarrow} X_{1} \stackrel{T_{1}}{\longrightarrow} X_{2} \stackrel{T_{2}}{\longrightarrow} \ldots \stackrel{T_{n}}{\longrightarrow} X_{n+1}
$$

splits (i.e. all operators $T_{i}: X_{i} \rightarrow \operatorname{Im} T_{i}$ for $i<n$ have continuous linear right inverses), whenever $X_{0}, X_{1}, \ldots, X_{n}$ are complemented subspaces of a nuclear space $\Lambda_{\infty}(\alpha)$.

(b) Every graded exact complex

$$
0 \longrightarrow X_{0} \stackrel{T_{0}}{\longrightarrow} X_{1} \stackrel{T_{1}}{\longrightarrow} X_{2} \stackrel{T_{2}}{\longrightarrow} \ldots \stackrel{T_{n}}{\longrightarrow} X_{n+1}
$$

graded splits (i.e. all operators $T_{i}: X_{i} \rightarrow \operatorname{Im} T_{i}$ for $i<n$ have graded linear right inverses), whenever $X_{0}, X_{1}, \ldots, X_{n}$ are graded complemented subspaces of $g-s^{\mathbb{N}}$.

(c) Every tamely exact complex

$$
0 \longrightarrow X_{0} \stackrel{T_{0}}{\longrightarrow} X_{1} \stackrel{T_{1}}{\longrightarrow} X_{2} \stackrel{T_{2}}{\longrightarrow} \ldots \stackrel{T_{n}}{\longrightarrow} X_{n+1}
$$

tamely splits (i.e. all operators $T_{i}: X_{i} \rightarrow \operatorname{Im} T_{i}$ for $i<n$ have tame continuous linear right inverses), whenever $X_{0}, X_{1}, \ldots, X_{n}$ are tamely complemented subspaces of a nuclear space $\Lambda_{0}(\alpha)$ (equipped with the standard sequence of norms).

Proof. (a): By Lemma 3.5 and [15, 29.4, 29.11, 30.1], the sequence

$$
0 \longrightarrow X_{0} \stackrel{T_{0}}{\longrightarrow} X_{1} \stackrel{T_{1}}{\longrightarrow} \operatorname{Im} T_{1} \longrightarrow 0
$$

splits, thus $\operatorname{Im} T_{1}$ is a complemented subspace of $\Lambda_{\infty}(\alpha)$. Repeating this procedure inductively for

$$
0 \longrightarrow X_{i+1} \stackrel{T_{i+1}}{\longrightarrow} \operatorname{Im} T_{i} \longrightarrow 0
$$

we get splitting of the whole sequence.

(b): By Lemma 3.5] and [5, Th. 4.2], the sequence

$$
0 \longrightarrow X_{0} \stackrel{T_{0}}{\longrightarrow} X_{1} \stackrel{T_{1}}{\longrightarrow} \operatorname{Im} T_{1} \longrightarrow 0
$$

graded splits, thus $\operatorname{Im} T_{1}$ is a graded complemented subspace of $g-s^{\mathbb{N}}$. Repeating this procedure inductively as in (a) we get splitting of the whole sequence.

(c): By [18, Cor. 6.3 (ii)], every tamely exact sequence of the form

$$
0 \rightarrow \Lambda_{0}(\alpha) \rightarrow G \rightarrow \Lambda_{0}(\beta) \rightarrow 0
$$

tamely splits whenever $\Lambda_{0}(\alpha), \Lambda_{0}(\beta), G$ are equipped with hilbertian norms, namely, on $\Lambda_{0}(\alpha)$ we take the norms

$$
\|x\|_{2, k}:=\left(\sum_{n \in \mathbb{N}}\left|x_{n}\right|^{2} e^{-\frac{2}{k} \alpha_{n}}\right)^{1 / 2}
$$

analogous norms are taken on $\Lambda_{0}(\beta)$. This holds because it is proved in [18, p. 157] that the above sequence satisfies the assumptions of [18, Th. 6.1]. Clearly, the assumptions are still satisfied if we take instead of $\Lambda_{0}(\alpha)$ a tame quotient of this space and instead of $\Lambda_{0}(\beta)$ a tame subspace of $\Lambda_{0}(\beta)$. By nuclearity, the sequences 
of norms $\left(\|\cdot\|_{2, n}\right)$ and $\left(\|\cdot\|_{n}\right)$ are tamely equivalent on $\Lambda_{0}(\alpha)$. Summarizing, the sequence

$$
0 \longrightarrow X_{0} \stackrel{T_{0}}{\longrightarrow} X_{1} \stackrel{T_{1}}{\longrightarrow} \operatorname{Im} T_{1} \longrightarrow 0
$$

tamely splits, thus $\operatorname{Im} T_{1}$ is a tame complemented subspace of $\Lambda_{0}(\alpha)$. Repeating this procedure inductively as in (a) we get (tame) splitting of the whole sequence.

Proof of Theorem 2.2. We will consider two cases.

1. $M$ is compact. If there exists an extension operator $S: C^{\infty}(X) \rightarrow C^{\infty}(M)$, then, by taking a covering of $M$ by local trivializations of $E \rightarrow M$ and proceeding as in the proof of Lemma 3.4, we see that there exists a continuous extension operator $T: C^{\infty}(X ; E) \rightarrow C^{\infty}(M ; E)$. Then the space $C^{\infty}(X ; E)$ is isomorphic to a complemented subspace of $C^{\infty}(M ; E)$. Analogously, $\Lambda_{k}^{\infty}(X ; E)$ is complemented in $\Lambda_{k}^{\infty}(M ; E)$. Using the same proof as in [24, Th. 2.3], we can show that $\Lambda_{k}^{\infty}(M ; E)$ is isomorphic to $s=\Lambda_{\infty}(\log n)$. This together with Theorem 2.1] shows that the assumptions of Lemma 3.6 (a) are satisfied.

2. $M$ is not compact. The exact sequences (3) and (4) are also exact when $X$ is substituted by any open subset of $X$ or $M$ (see Theorem 2.1 in the smooth category). Therefore both sequences are graded exact (see [5, Section 2 and 1]). By Lemma 3.4 the assumptions of Lemma 3.6 (b) are satisfied.

Proof of Theorem 2.3. Since $E$ is trivial, then

$$
\Lambda_{p}^{\text {Stein }}(M ; E) \simeq H(M)^{l},
$$

with some $l$. If $X=M$ satisfies the strong Liouville property [25] Th. 0.3], then

$$
H(M)^{l} \simeq \Lambda_{\infty}\left(n^{1 / d}\right)^{l} \simeq \Lambda_{\infty}\left(n^{1 / d}\right) .
$$

The splitting of exact sequences (1) and (2) follows from Lemma 3.6 (a).

Let us assume that $M$ is strongly pseudoconvex. By Lemma $3.3\left(H(M)^{l}\right.$, $\left.\left(\|\cdot\|_{n}\right)_{n \in \mathbb{N}}\right)$ is tamely isomorphic to $\left(\Lambda_{0}\left(n^{1 / d}\right)\right)^{l} \simeq \Lambda_{0}\left(n^{1 / d}\right)$.

Since the sequences (1) and (2) are also exact when $M$ is substituted by any set $\Omega_{c}$ from Lemma 3.3 (see Theorem 2.1 in the Stein category), therefore both sequences are tamely exact (see the form of seminorms on $H(M)^{l}$ !). By Lemma 3.6 (c), both complexes split.

Remark. Note that the crucial role in our proofs is played by the Splitting Theorem of Vogt [15 30.1].

\section{ACKNOWLEDGEMENT}

The authors wish to thank Misha Zhitomirskii for discussions which led to the division problems considered here, and Michael Langenbruch for pointing out some references.

\section{REFERENCES}

[1] E. Bierstone, P. Milman, Local analytic invariants and splitting theorems in differential analysis, Israel J. Math. 60 (1987), 257-280. MR 89g:58021

[2] E. Bierstone, P. Milman, Geometric and differential properties of subanalytic sets, Ann. Math. 147 (1998), 731-785. MR 2000c:32027

[3] E. Bierstone, G. W. Schwartz, Continuous linear division and extension of $C^{\infty}$ functions, Duke Math. J. 50 (1983), 233-271. MR 86b:32010

[4] H. Cartan, Variétés analytiques réelles et variétés analytiques complexes, Bull. Soc. Math. France 85 (1957), 77-99. MR 20:1339 
[5] P. Domański, D. Vogt, A splitting theorem for the space of smooth functions, J. Funct. Anal. 153 (1998), 203-248. MR 99b:46001

[6] P. Domański, D. Vogt, A splitting theory for the space of distributions, Studia Math. 140 (2000), 57-77. MR 2001e:46125

[7] P. Domański, D. Vogt, Distributional complexes split for positive dimensions, J. reine angew. Math. 522 (2000), 63-79. MR 2001g:46090

[8] D. Eisenbud, Commutative Algebra, Springer Verlag, 1994. MR 97a:13001

[9] L. Frerick, Extension operators for spaces of infinitely differentiable Whitney functions, Habilitationsschrift, Wuppertal, 2001.

[10] P. Griffiths, J. Harris, Principles of Algebraic Geometry, Wiley and Sons, New York, 1978. MR 80b:14001

[11] F. Guaraldo, P. Macri, A. Tancredi, Topics on Real Analytic Spaces, Vieweg, Braunschweig, 1986. MR 90j:32001

[12] B. Jakubczyk, M. Zhitomirskii, Local reduction theorems and invariants for singular contact structures, Ann. Inst. Fourier 51 (2001), 237-295. MR 2002c:58001

[13] B. Jakubczyk, M. Zhitomirskii, Distributions of corank 1 and their characteristic vector fields, Trans. Amer. Math. Soc., to appear.

[14] B. Malgrange, Ideals of Differentiable Functions, Oxford University Press, Oxford, 1966. MR 35:3446

[15] R. Meise, D. Vogt, Introduction to Functional Analysis, Clarendon Press, Oxford, 1997. MR 98g:46001

[16] R. Moussu, Sur l'existence d'intégrales premières pour un germe de forme de Pfaff, Ann. Inst. Fourier 26 (1976), 171-220. MR 54:3737

[17] R. Narasimhan, Introduction to the Theory of Analytic Spaces, Lecture Notes in Math. 25, Springer, Berlin, 1966. MR 36:428

[18] M. Poppenberg, D. Vogt, A tame splitting theorem for exact sequences of Fréchet spaces, Math. Z. 219 (1995), 141-161. MR 96h:46109

[19] S. Rolewicz, Metric Linear Spaces, PWN - Polish Scientific Publisher, Warszawa, 1984. MR 55:10993 (review of 1972 edition)

[20] J.M. Ruiz, The Basic Theory of Power Series, Advanced Lectures in Mathematics, Vieveg, Wiesbaden, 1993. MR 94i:13012

[21] J.-P. Serre, Faisceaux algébriques cohérents, Ann. Math. 69 (1955), 197-278. MR 16:953c

[22] M. Tidten, Fortsetzungen von $C^{\infty}$-Funktionen, welche auf einer abgeschlossenen Menge in $\mathbb{R}^{n}$ definiert sind, Manuscripta Math. 27 (1979), 291-312. MR 80k:58016

[23] J. C. Tougeron, Ideaux de Fonctions Differentiables, Springer, Berlin, 1972. MR 55:13472

[24] D. Vogt, Sequence space representations of spaces of test functions and distributions, in: Functional Analysis, Holomorphy and Approximation Theory, G. L. Zapata (ed.), Lecture Notes Pure Appl. Math. 83, Marcel Dekker, New York, 1983. MR 84f:46048

[25] V. P. Zaharjuta, Spaces of analytic functions and complex potential theory, in: Linear Topological Spaces and Complex Analysis 1, A. Aytuna (ed.), METU-TÜBİTAK, Ankara, 1994, pp. 74-146. MR 97d:46002

Faculty of Mathematics and Computer Science, A. Mickiewicz University and Institute of Mathematics (Poznań Branch), Polish Academy of Sciences, Umultowska 87, 61-614 Poznań, Poland

E-mail address: domanski@math.amu.edu.pl

Institute of Applied Mathematics, Warsaw University, Ul. Banacha 2, 02-097 Warszawa, Poland On leave from: Institute of Mathematics, Polish Academy of Sciences, WarszaWa, Poland

E-mail address: B.Jakubczyk@impan.gov.pl 